\title{
Chromaticity Tracking Using a Phase Modulation Technique *
}

\author{
C.Y. $\operatorname{Tan}^{\dagger}$, Fermilab, Batavia, IL 60510, USA
}

\section{Abstract}

In the classical chromaticity measurement technique, chromaticity is measured by measuring the change in betatron tune as the RF frequency is varied. This paper will describe a novel way of measuring chromaticity: we will phase modulate the RF with a known sine wave and then phase demodulate the betatron frequency. The result is a line in Fourier space which corresponds to the frequency of our sine wave modulation. The peak of this sine wave is proportional to chromaticity. For this technique to work, a tune tracker PLL system is required because it supplies the betatron carrier frequency. This method has been tested in the Tevatron and we will show the results here.

\section{INTRODUCTION}

In the classical chromaticity measurement technique, chromaticity is measured by measuring the change in betatron tune as the the RF frequency is varied. In this paper, we will describe a novel way invented by D. McGinnis[1] for measuring chromaticity: the beam is phase modulated longitudinally by the RF with a known sine wave and then the chromaticity is obtained by phase demodulating the betatron frequency carried transversely by the beam. Unfortunately, early experiments with this method did not yield good results because the betatron tune drifts in the Tevatron and the vector signal analyzer used as a phase demodulator would lose lock and stop reporting chromaticity. So, this method was abandoned in favour of other methods.

Fortunately, as time passed, a tune tracker (TT) phase locked loop (PLL) system[2] became operational in the Tevatron and McGinnis' method could be resurrected because when the tune tracker is locked to the betatron tune and tracking, it supplies the carrier frequency from which phase demodulation can be carried out.

\section{THEORY}

McGinnis' method starts with the modulation of the RF frequency $\dot{\phi}_{\mathrm{RF}}(t)$ with a sinusoid

$$
\dot{\phi}_{\mathrm{RF}}(t)=\omega_{\mathrm{RF}}+\Delta \phi_{\mathrm{mod}} \times \Omega_{\mathrm{mod}} \cos \left(\Omega_{\bmod } t\right)
$$

where $\omega_{\mathrm{RF}}$ is the RF frequency when there is no modulation, $\Delta \phi_{\text {mod }}$ is the amplitude of the phase modulation and $\Omega_{\text {mod }}$ is the RF phase modulation. It can be shown that

\footnotetext{
* Work supported by Fermi Research Alliance, LLC under Contract No. DE-AC02-07CH1 1359 with the United States Department of Energy.

†cytan@fnal.gov
}

the betatron phase $\phi_{\beta}$ will then be modulated and its amplitude is linearly dependent on the chromaticity $\chi$. The relationship between $\phi_{\beta}$ to $\chi$ is

$$
\begin{aligned}
\phi_{\beta}(t) & =\omega_{\mathrm{rev}} Q_{0} t+\frac{\Delta \phi_{\mathrm{mod}}}{h}\left(Q_{0}-\frac{\chi}{\eta}\right) \sin \Omega_{\bmod } t \\
& +\Delta \phi_{s}\left(Q_{0}-\frac{\chi}{\eta}\right) \sin \left(\Omega_{s} t+\theta_{s}\right)+\phi_{\beta_{0}}
\end{aligned}
$$

where $\omega_{\text {rev }}$ is the revolution frequency, $Q_{0}$ is the betatron tune, $h$ is the harmonic number, $\eta$ is the slip factor, $\Delta \phi_{s}$ is the amplitude of the synchrotron phase modulation, $\Omega_{s}$ is the synchrotron frequency, $\theta_{s}$ is the synchrotron phase and $\phi_{\beta_{0}}$ is the betatron phase at $t=0$.

In reality, we measure the betatron tune by taking the difference of the image current $I_{\Delta}$ between two plates i.e. at a pickup. This difference is

$$
\begin{aligned}
I_{\Delta}(t)= & \omega_{\mathrm{rev}} q_{b} \frac{A}{\sqrt{\epsilon}} \cos \left[\phi_{\beta}(t)\right] \\
& \times \sum_{n=-\infty}^{\infty} \delta\left(\phi_{r}(t)-2 n \pi-\phi_{r}(0)\right) \\
= & \frac{\omega_{\mathrm{rev}} q_{b}}{2} \frac{A}{\sqrt{\epsilon}} \sum_{k=0}^{\infty} C_{k} \sum_{m=-\infty}^{\infty} \sum_{n=-\infty}^{\infty} J_{m}\left(Y_{+}\right) J_{n}\left(Z_{+}\right) \\
& \times \cos \left(\omega_{k m n}^{+} t+\psi_{+}\right) \\
& +\frac{\omega_{\mathrm{rev}} q_{b}}{2} \frac{A}{\sqrt{\epsilon}} \sum_{k=0}^{\infty} C_{k} \sum_{m=-\infty}^{\infty} \sum_{n=-\infty}^{\infty} \\
& J_{m}\left(Y_{-}\right) J_{n}\left(Z_{-}\right) \cos \left(\omega_{k m n}^{-} t+\psi_{-}\right)
\end{aligned}
$$

where

$$
\begin{aligned}
\phi_{r}(t) & =\omega_{\mathrm{rev}} t+\frac{\Delta \phi_{\mathrm{mod}}}{h} \sin \left(\Omega_{\mathrm{mod}} t\right) \\
& +\Delta \phi_{s} \sin \left(\Omega_{s} t+\theta_{s}\right)
\end{aligned}
$$

and $q_{b}$ is the charge of the bunch, $A$ is the betatron amplitude at the pickup, $\epsilon$ is the transverse emittance, $C_{0}=1 / 2 \pi, C_{k}=1 / \pi, Y_{ \pm}=\left(k \pm Q_{0} \mp \frac{\chi}{\eta}\right) \Delta \phi_{s}$, $Z_{ \pm}=\left(k \pm Q_{0} \mp \frac{\chi}{\eta}\right) \frac{\Delta \phi_{\text {mod }}}{h}, \psi_{ \pm}=k \phi_{r}(0) \pm \phi_{\beta}(0)$, and $\omega_{k m n}^{ \pm}=\left(k \pm Q_{0}\right) \omega_{\mathrm{rev}}+m \Omega_{s}+n \Omega_{\mathrm{mod}}$.

Therefore, Eq. (3) shows that at a given betatron mode $k$, there are an infinite number of modes $m$ associated with the synchrotron frequency. Around any mode $(k, m)$, there are an infinite number of modes $n$ associated with the 
RF modulation. In general, the TT will just lock to one synchrotron sideband. Suppose the synchrotron line that the TT locks to is mode $(k, m,+)$, then its mode frequency is $\omega_{k m}^{+}=\left(k+Q_{0}\right) \omega_{\text {rev }}+m \Omega_{s}$. The spectrum of modes associated with the RF modulation in temporal space is

$$
\begin{gathered}
I_{\Delta}(t ; k, m,+) \propto \sum_{n=-\infty}^{\infty} J_{n}\left(Z_{+}\right) \\
\times \cos \left(\omega_{k m}^{+} t+n \Omega_{\bmod } t+\psi_{+}\right) \\
=\cos \left(\omega_{k m}^{+} t+Z_{+} \sin \Omega_{\bmod } t+\psi_{+}\right)
\end{gathered}
$$

This result shows that the carrier frequency is $\omega_{k m}^{+}$and the phase is modulated by $Z_{+} \sin \Omega_{\bmod } t$. More importantly, it also shows that we can phase demodulate using any synchrotron line as the carrier to obtain $\chi$ because the peak amplitude of the modulation $Z_{+}$is independent of both $m$ and the amplitude of the synchrotron line. A similar argument will show that this is also true for the synchrotron line $(k, m,-)$ where $Z_{-}$is the peak modulation amplitude. Therefore, $\chi$ can be calculated once we identify $(k, \pm)$.

\section{EXPERIMENT}

The experimental setup is shown in Figure 1. The TT provides the carrier frequency for the phase detector when it is locked to the betatron tune or its synchrotron sidebands. The phase detector demodulates the phase modulation carried by the beam w.r.t. the carrier frequency. For our setup in the Tevatron, $k-Q_{0} \approx 448.5, h=1113$, $\eta=0.0029$. The RF modulation frequency $\Omega_{\bmod }=$ $(2 \pi \times 23) \mathrm{s}^{-1}$ was chosen to be smaller than the synchrotron frequency which is $84 \mathrm{~Hz}$ at $150 \mathrm{GeV}$ and $34 \mathrm{~Hz}$ at $980 \mathrm{GeV}$.

\section{Hardware Phase Detector}

The hardware phase detector is built around a Cyclone NIOS II processor and $2 \times 14$ analogue to digital converters (ADCs) and digital to analogue converters (DACs). Internal to the Cyclone are two hardware Hilbert transformers which phase shift the input betatron signal and the reference signal from the TT by $\pi / 2$ so that an in phase and quadrature signal can be produced. This signal is processed with the NIOS II by software to produce a phase. The amplitude $Z_{-}$of the phase oscillations is calculated using a sliding Goertzel algorithm. The result is sent back to the control system via ethernet where the chromaticity is calculated. A picture of the hardware phase detector is shown in Figure 2.

\section{Results}

The results with $\Delta \phi_{\bmod }=4^{\circ}$ and $8^{\circ}$ are shown in Figure 3 for one bunch of uncoalesced beam at $150 \mathrm{GeV}$.
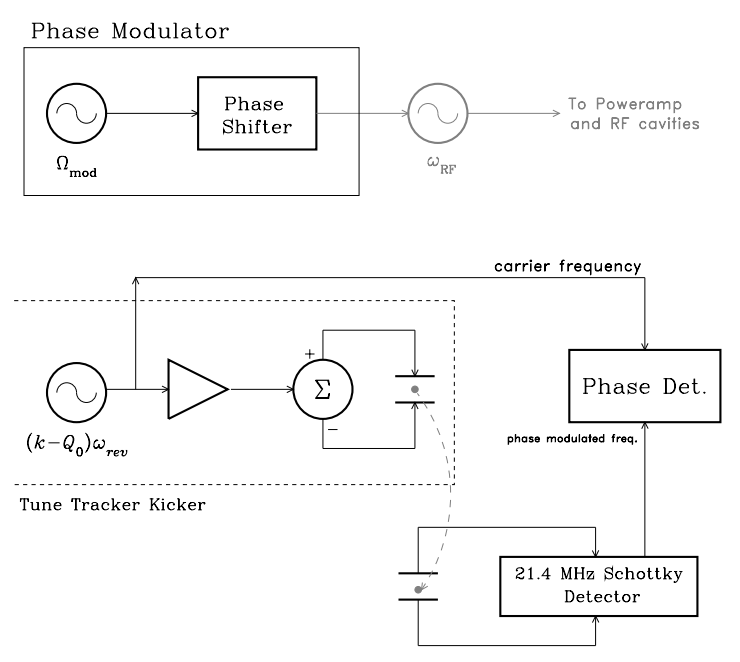

Figure 1: The experimental setup is shown here. The phase detector, shown in Figure 2, detects the phase modulation w.r.t. the carrier frequency given by the TT.

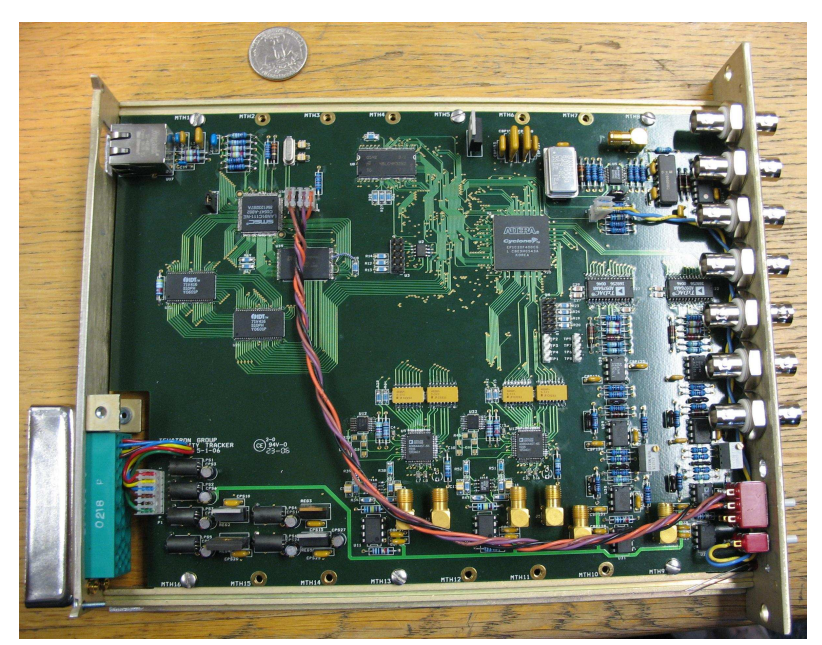

Figure 2: This is the phase detector board which is built around a Cyclone NIOS II processor. It runs at $50 \mathrm{MHz}$ with $2 \times 14$ bit ADCs and DACs.

The horizontal chromaticity is changed by varying a calibrated knob "CXINJ". It is clear that the chromaticity is well tracked when $\Delta \phi_{\bmod }=8^{\circ}$, but at $\Delta \phi_{\bmod }=4^{\circ}$, it is noisier. However even at $\Delta \phi_{\bmod }=8^{\circ}$, the error is \pm 1 unit. The source of this error will be discussed in the next section.

It is also interesting to note that there is a small change in the betatron tune when CXINJ is changed. See Figure 4 box (a). In principle, this should not affect the chromaticity measurement, however, we have discovered some problems which need to be addressed which will be discussed in the next subsection. 

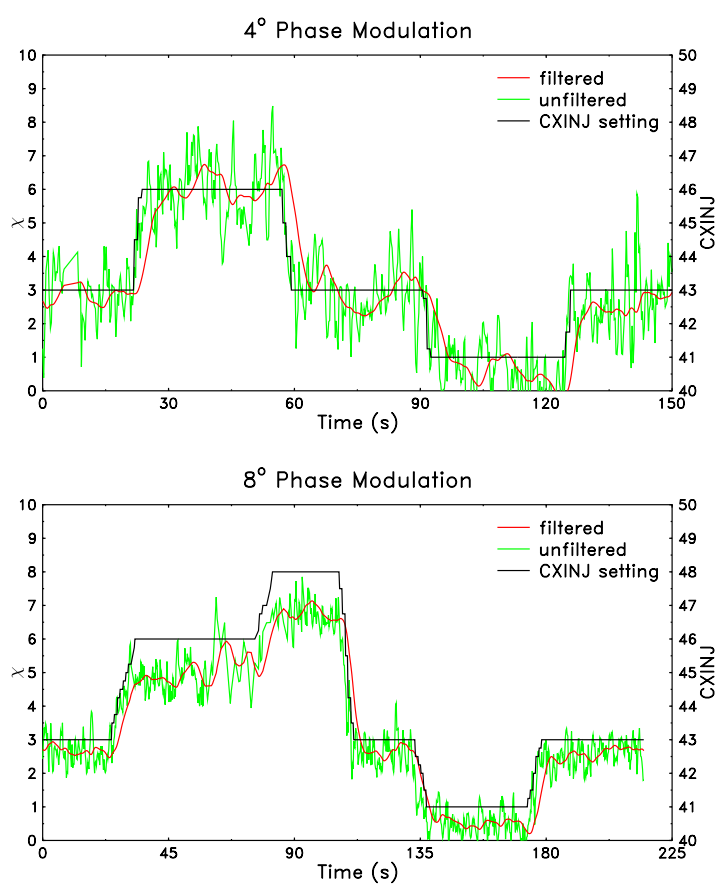

Figure 3: The difference between $\Delta \phi_{\bmod }=4^{\circ}$ and $8^{\circ}$ is quite startling. An $8^{\circ}$ phase modulation gives very good agreement between the calibrated knob CXINJ and the tracked chromaticity (red) while a $4^{\circ}$ phase modulation is much noisier and does not track as well.

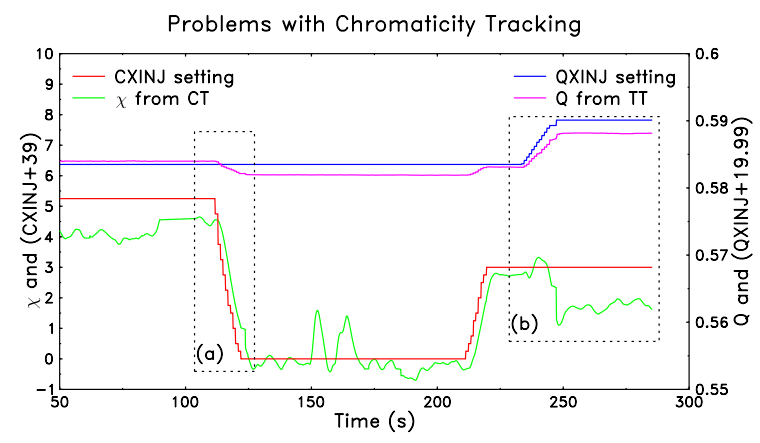

Figure 4: Box (b) of this figure shows the problem we have right now with the CT: CT tracks the changes in QXINJ when CXINJ is held constant. Box (a) shows small tune changes when CXINJ is changed.

\section{Problems}

Although the chromaticity tracker (CT) seems to follow CXINJ well, we have observed that the CT tracks changes of betatron tune despite CXINJ being held constant. See Figure 4 box (b). When the betatron tune is changed with QXINJ, the TT tracks this change perfectly but it is clear that the CT tracks this change and decreases by 1.5 units. We suspect that the CT is responding to changes in the input betatron signal when the TT responds to changes in

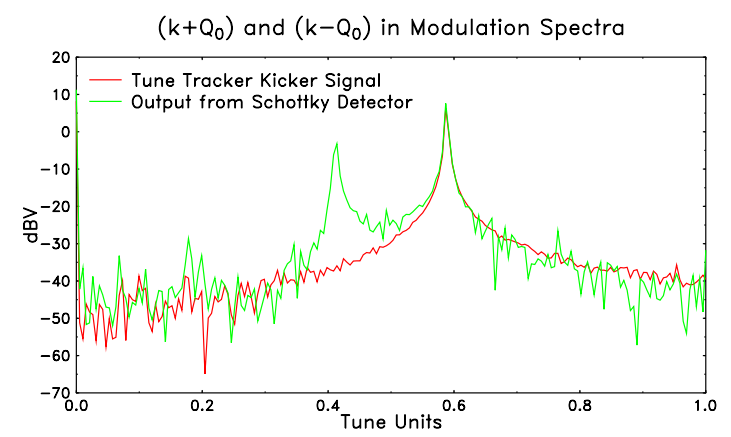

Figure 5: The two peaks in the output of the Schottky detector creates a problem for the phase detector. The lower peak is the $\left(k+Q_{0}\right)$ line which we did not take care of in the original programming. The red peak $\left(k-Q_{0}\right)$ from the tune tracker kicker is coincident with the upper peak of the Schottky detector.

QXINJ. The source of this problem has been traced to an extra frequency that has not been taken into account: the $\left(k+Q_{0}\right)$ line. See Figure 5. We have found a fix for this problem by adding filters to the phase detector. This has been demonstrated to work well on the bench. Machine studies will be performed at the earliest opportunity to check that this fix works.

\section{CONCLUSION}

We have found that this method allows us to track chromaticity changes of \pm 1 units in the Tevatron at $150 \mathrm{GeV}$. Unfortunately, we have discovered that the CT tracks changes in betatron tune as well. We found that the cause of this problem is because the $\left(k+Q_{0}\right)$ line was not taken into account at the phase detector. A fix has been demonstrated to work well on the bench and will be tested with real beam soon.

\section{REFERENCES}

[1] D. McGinnis, "Chromaticity Measurements using Phase Modulated RF and Vector Signal Analyzers", PBAR Note 656, Fermilab, 2001.

[2] C.Y. Tan, "The Tevatron Tune Tracker PLL — Theory, Implementation and Measurements", TM-2275, Fermilab, 2004. 